\title{
Avaliação do processo psicoterápico: levantamento de técnicas e instrumentos
}

\section{Assessment of the psychotherapeutic process: survey of techniques and instruments}

\author{
Ana Carolina Zuanazzi (orcid.org/0000-0003-1649-2372)'1 \\ Thaline da Cunha Moreira (orcid.org/0000-0002-6484-8583) ${ }^{2}$ \\ Francine Náthalie Ferraresi Rodrigues Queluz (orcid.org/0000-0002-8869-6879) 3 \\ Anna Elisa Villemor-Amaral (orcid.org/0000-0002-1815-8194)4
}

\begin{abstract}
Resumo
Um dos aspectos mais estudados em intervenções psicoterápicas são os procedimentos empregados em psicoterapia que resultam em melhores benefícios ao paciente em um menor espaço de tempo, ou seja, a avaliação da eficiência do processo psicoterápico, prezando-se não apenas pela ética com o paciente, mas também pela redução de gastos com tratamentos. Diante disso, o objetivo deste estudo foi levantar quais os principais instrumentos utilizados com essa finalidade. Por meio de busca sistematizada de literatura, foram resgatados 5.938 artigos que, após triagem por meio de critérios de inclusão e exclusão, resultaram em um conjunto de 157 estudos analisados em sua íntegra, o que permitiu identificar 114 instrumentos e técnicas. Após a apresentação dos instrumentos mais recorrentes na literatura, algumas considerações foram tecidas a respeito do panorama atual de avaliação de processos psicoterapêuticos, como, por exemplo, a escassez de instrumentos brasileiros e as contribuições desta pesquisa para futuros estudos nessa temática.
\end{abstract}

Palavras-chave: Psicoterapia. Aliança terapêutica. Processo psicoterapêutico. Medidas. Avaliação psicológica.

\begin{abstract}
One of the most studied aspects in psychotherapeutic interventions are the procedures used in psychotherapy that result in better patient benefits in a shorter period, the evaluation of psychotherapeutic process's efficiency, considering ethics toward the patient, and reducing treatment costs. This study aimed to identify the main instruments used for this purpose. Through a systematized review of literature, 5,938 articles were found, which, after screening by inclusion and exclusion criteria, resulted in a set of 157 studies and the identification of 114 instruments and techniques. After presenting the most recurrent instruments in the literature, some considerations
\end{abstract}

\footnotetext{
${ }^{1}$ Universidade São Francisco, São Paulo, Brasil. E-mail: anacarolina.zf@gmail.com

${ }^{2}$ Universidade São Francisco, São Paulo, Brasil. E-mail: thacmoreira@gmail.com

${ }^{3}$ Universidade São Francisco, São Paulo, Brasil. E-mail: francine.queluz@gmail.com

${ }^{4}$ Universidade São Francisco, São Paulo, Brasil. E-mail: aevillemor@gmail.com
} 
were made regarding the current panorama of evaluation of psychotherapeutic processes, such as the scarcity of Brazilian instruments and the contributions of this research to future studies in this subject.

Keywords: Psychotherapy. Therapeutic alliance. Psychotherapeutic process. Measures. Psychological assessment.

Avaliar a eficácia e a eficiência de processos psicoterápicos é um dos principais focos de estudos sobre intervenções psicológicas. Verificar quais métodos são mais benéficos e quais condições levam aos melhores resultados, considerando aspectos éticos, sociais, políticos e econômicos envolvidos na condução de tratamentos psicológicos, é muito relevante, dada a diversidade de teorias e técnicas de intervenção em uso no Brasil e no mundo (Brum et al., 2012).

Avaliações adequadas desse processo permitem a observação de quais são as melhores opções terapêuticas, em termos de eficácia e eficiência, prezando pela ética no trato com pacientes, possibilitando o atendimento de um maior contingente de pessoas e diminuindo os custos, uma vez que tratamentos não eficazes resultam em prejuízos financeiros e de tempo, e requerem muita energia por parte de terapeutas e clientes. Devido a esses impactos, pesquisadores em diferentes contextos começaram a investigar o que e como avaliar resultados de processos psicoterápicos (Couto, 2015; Drozd \& Goldfried, 1996; Duncan, 2012; Peuker, Habigzang, Koller, \& Araujo, 2009).

Existem diferentes abordagens na avaliação desses resultados, desde métodos mais simplificados até os mais integrativos (Drozd \& Goldfried, 1996). As duas principais abordagens indicadas por Brum et al. (2012) são a avaliação de eficácia e a avaliação do processo psicoterapêutico enquanto ação em desenvolvimento. O primeiro tipo de avaliação, indicado por Ferreira e Yoshida (2004) como mais frequentemente utilizado em pesquisa no contexto de psicoterapia breve, busca, basicamente, verificar os resultados, enquanto o segundo se propõe a levantar os indicadores sobre o processo psicoterapêutico como um todo que o tornam uma experiência positiva para as pessoas envolvidas, ou seja, sua eficiência.

A primeira abordagem, avaliação da eficácia, geralmente compreende uma amostra de pessoas com transtorno ou sintomatologia semelhantes, na qual são aplicados 
instrumentos que mensuram determinadas características ou sintomas. Uma intervenção padronizada é conduzida e, ao final desta, aplica-se o mesmo instrumento ou algum outro com objetivo similar. Compara-se a sintomatologia pontuada antes e após a intervenção, verificando seus efeitos (Brum et al., 2012).

Uma das principais características desse modelo é o controle do maior número possível de variáveis. Por exemplo, é comum a existência de ao menos um grupo controle (com pessoas que não passarão pela intervenção), número fixo de sessões, conduta padronizada por parte do(s) terapeuta(s) e similaridade de sintomas dos participantes que farão parte do grupo interventivo (Brum et al., 2012; Drozd \& Goldfried, 1996). Contudo, algumas críticas são dirigidas a esse método de avaliação de psicoterapia devido à alta especificidade dos tratamentos, se distanciando de condições ecológicas reais aplicáveis. Aliado a isso, argumenta-se que esses métodos, em muitos casos, não acessam aspectos individuais tanto do terapeuta quanto do paciente (Seligman, 1995), fatores que têm sido considerados fundamentais nesse campo (Lutz, De Jong, \& Rubel, 2015).

Já a avaliação do processo compreende a psicoterapia como um movimento contínuo e não como um produto final, levando em consideração a promoção de mudanças ao longo do tempo em diferentes aspectos, como sentimentos, pensamentos e comportamentos, além da percepção por parte do sujeito de tais mudanças. Avaliam-se aspectos que dizem respeito à relação entre terapeuta e cliente, tentando identificar possíveis estratégias nessa relação que sejam benéficas ao cliente (aliança terapêutica, por exemplo). Também é avaliada a percepção dos clientes em termos de motivação, expectativa e percepção da eficiência terapêutica (Arnow et al., 2013; Brum et al., 2012; Duncan, 2012; Lutz et al., 2015).

Silveira e Wiethaeuper (2003) indicaram que, na década de 2000, havia poucos estudos nacionais direcionados à investigação sobre o processo psicoterapêutico, sendo a maioria com ênfase em métodos qualitativos. As pesquisas caminhavam em direção contrária às internacionais, que se respaldavam em delineamentos quantitativos, objetivando comparações entre grupos e generalização de resultados. Dados semelhantes foram encontrados por Serralta, Nunes e Eizirik (2007) e Yoshida, Santeiro, Moraes e Rocha (2005), 
além da indicação de carência de instrumentos próprios para a avaliação de mudanças em psicoterapia (Carvalho \& Rocha, 2009).

No contexto internacional, embora haja ênfase na avaliação dos resultados, há também publicações sobre avaliação do processo terapêutico (Cassel, Sanchez, Campezatto, \& Nunes, 2015). Diante disso, e dada a diversidade de instrumentos existentes internacionalmente e a necessidade de avaliação do processo terapêutico de forma rigorosa, considerando também a validade e precisão de instrumentos, este estudo teve como objetivo mapear, por meio de revisão de literatura, instrumentos que avaliam a percepção de eficiência de processos psicoterapêuticos, tanto no contexto nacional como internacional.

\section{Método}

O levantamento bibliográfico seguiu o modelo rapid review (Harker \& Kleijnen, 2012). Esse método de revisão se propõe à busca operacionalizada em bases de dados seguindo critérios rigorosos de inclusão e exclusão, com a finalidade de atingir o(s) objetivo(s) proposto(s). A rapid review não segue todos os procedimentos de uma revisão sistemática uma vez que, após a extração do material a ser analisado, não computa pontuações baseadas em check-lists. Apesar disso, esse método de revisão é considerado suficientemente rigoroso para análise de grande quantidade de material publicado sobre um tema alvo.

Tendo como base o método de revisão aqui proposto, foi feita a procura eletrônica de artigos nas bases de dados Psyclnfo, Lilacs e Science, sem restrição de tempo, até junho de 2018. Tais bases foram escolhidas por reunirem artigos submetidos a uma revisão por pares. Os descritores utilizados e suas configurações para a pesquisa foram os seguintes: Psyclnfo "(test OR scale OR method OR instrument) AND ("psychotherapeutic assessment" OR interventions OR treatment OR "psychotherapeutic process") AND (efficacy OR effectiveness OR outcome OR "psychotherapy outcome" OR "psychotherapy change" OR "treatment outcome")". Lilacs "avaliação AND psicoterapia AND processo OR resultado". Science "("Psychologic Tests" OR "Psychologic Test" OR "Test, Psychologic" OR "Psychological Test") AND ("Psychotherapies" OR "Psychotherapists" OR "Psychotherapist" OR "Clinical 
Psychotherapists" OR "Clinical Psychotherapist" OR "Psychotherapists, Clinical") AND ("outcome, treatment" OR " clinical effectiveness" OR "clinical effectiveness" OR "effectiveness, clinical" OR "effectiveness, clinical" OR "patient-relevant outcome" OR "outcome, patient-relevant" OR "outcomes, patient-relevant" OR "patient relevant outcome" OR "clinical efficacy" OR "efficacy" OR "treatment effectiveness" OR "effectiveness, treatment" OR "treatment efficacy" OR "Efficacy, Treatment")".

Para a seleção dos artigos, os seguintes critérios de inclusão foram aplicados: (1) o artigo deveria pertencer a um periódico indexado e revisado por pares, (2) utilizar instrumentos específicos para a avaliação do processo terapêutico e (3) apresentar os descritores desta revisão nas palavras-chave, título e/ou no resumo. Foram excluídos do presente estudo artigos que não utilizaram um instrumento que avaliasse especificamente o processo terapêutico ou que concomitantemente não foram revisados por pares, tal como livros, trabalhos acadêmicos, apresentações em congressos e outros formatos diferentes de artigos. Os critérios de inclusão e exclusão foram os mesmos em todas as etapas de seleção.

A partir dos critérios de inclusão e exclusão, realizou-se um levantamento preliminar, por meio da leitura dos resumos de cada pesquisa. Essa fase foi realizada por dois juízes, autores do presente estudo, a fim de se obter uma revisão por duplas dos critérios de seleção adotados. Os juízes conferiram os resumos e selecionaram aqueles em que os critérios de inclusão e exclusão foram precisamente atendidos (conforme indicado anteriormente). Os artigos selecionados foram lidos na íntegra e, com isso, uma nova seleção foi realizada, formalizando, assim, o material de análise desta revisão.

No total, foram coletados, inicialmente, 5.938 artigos científicos e, após a exclusão de artigos duplicados, os resumos foram lidos e avaliados pelos pares de juízes. Com isso, foram excluídos 5.283 artigos que não atenderam aos critérios de inclusão explicitados anteriormente, sendo selecionado um total de 655 para leitura completa. Após a leitura completa desses artigos, foram selecionados 157, que fizeram parte do escopo final deste estudo por atingirem todos os critérios de inclusão e exclusão, conforme apresentado anteriormente. Na Figura 1, apresenta-se um fluxograma do processo de busca e seleção dos artigos, bem como os estudos eliminados conforme os critérios de inclusão e exclusão já mencionados. 
Os artigos selecionados foram categorizados com a finalidade de recolher o maior número de informações a respeito deles. Assim, os dados foram tabulados em uma planilha na qual foram reunidas as informações quanto ao ano de publicação, periódico e nacionalidade dos autores. As frequências desses dados foram levantadas pela estatística descritiva, por meio do software Statistical Package for the Social Sciences (SPSS) versão 21. Em seguida, foram selecionados os instrumentos que apareceram com maior frequência e foi realizada uma breve descrição dos mesmos, com o intuito de se verificar o que está sendo mais utilizado na área e suas principais características.

Figura 1 Fluxograma do processo de busca e seleção de artigos

\section{Resultados}

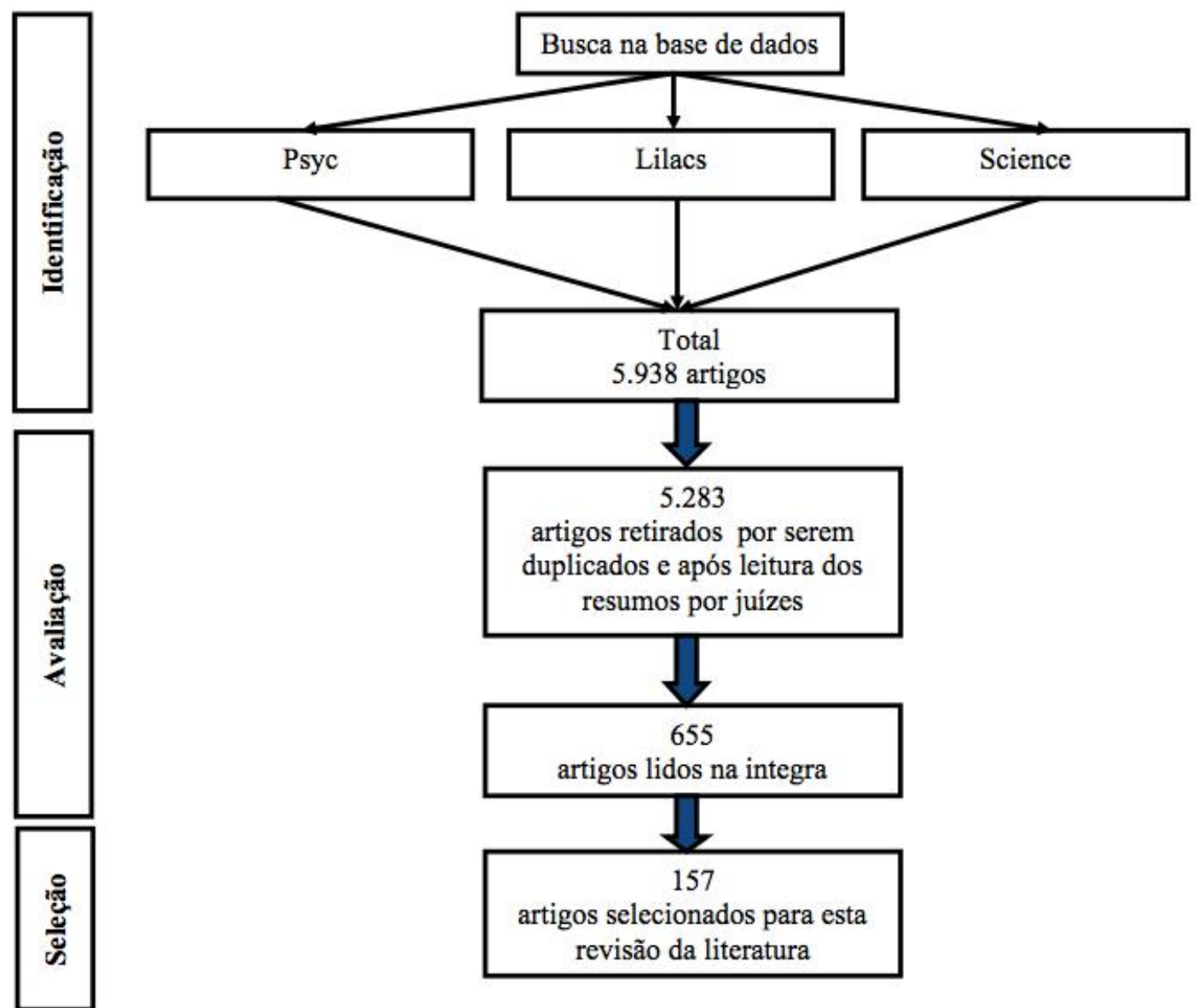


Verifica-se que, no que diz respeito ao ano de publicação, há estudos que utilizaram instrumentos para avaliar a eficácia terapêutica, publicados desde 1965. Ao longo dos anos, pelo menos um a dois artigos foram publicados nas bases de dados pesquisadas, sendo observado um intervalo de, no máximo, três anos entre eles. Comparativamente a 1965, houve aumento de publicações que passou a acontecer a partir de $2009(n=11)$ e 2012 ( $n=15)$, com leve redução nos anos de $2014(n=10), 2015(n=10)$ e importante aumento no ano de $2016(n=21)$. Dessa forma, foi verificado que somente no ano de 2016 é que o tema de fato se destacou, quando comparado com todos os anos desde 1965. Ainda é possível verificar que, no ano de 2017 , apenas três publicações foram encontradas e, até o momento da realização desse levantamento, foi encontrado um total de dez publicações para 2018. Esses dados informam que no ano de 2017 houve uma queda significativa nas publicações com essa temática.

No que se refere aos periódicos em que se encontram os estudos selecionados, houve uma grande variedade de revistas científicas, sendo que, do total (157), apenas sete eram brasileiras, são elas: Psico USF, Avaliação Psicológica, Psicologia Clínica, Psicologia Teoria e Pesquisa, Estudos de Psicologia, Revista Psiquiatria do Rio Grande do Sul e Terapia Psicológica. Com exceção da Psico USF $(n=4)$ e da Revista Psiquiatria do Rio Grande do Sul e Estudos de Psicologia ( $n=2$, cada uma), as demais revistas tiveram apenas uma publicação. Entre as internacionais, as que tiveram o maior número de publicações com o tema foram: Journal of Consulting and Clinical Psychology $(\mathrm{n}=44)$, seguido do Journal of Counseling Psychology $(\mathrm{n}=19)$, Psychoterapy $(\mathrm{n}=16)$, Psychotherapy: Theory, Research, Practice, Training $(n=14)$ e Psychological Assessment $(n=10)$. Vale ressaltar que, em termos de frequência de publicação, como se pode observar, há poucos estudos brasileiros.

Considerando que a maior parte dos estudos foi publicada em revistas internacionais, buscou-se também, para esta pesquisa, avaliar a nacionalidade dos autores, com a finalidade de verificar o local em que se encontram os polos que buscam avaliar a eficácia de um processo terapêutico. Observou-se artigos decorrentes de 16 nacionalidades diferentes, com a grande parte dos autores sendo proveniente de instituições localizadas nos Estados Unidos da América (EUA; $n=110)$, seguidos do Brasil $(n=11)$, Canadá $(n=5)$ e Chile $(n=5)$. 
Atendendo mais especificamente ao objetivo do presente estudo, foram levantados os instrumentos que tinham como objetivo avaliar a eficácia do processo terapêutico. Foram incluídos tanto aqueles com base estruturada (psicométricos) como os semiestruturados/não estruturados (e.g., entrevistas, vídeos, etc.). Para contabilização dos instrumentos, foram incluídos todos os que foram apresentados nos artigos que atendiam ao objetivo da pesquisa. Um total de 114 instrumentos foi mapeado (considerando que nos estudos, muitas vezes, eram utilizados até dois instrumentos para avaliação do processo psicoterapêutico), sendo 98 estruturados e 16 semiestruturados/não estruturados. Nessa categoria dos semiestruturados/não estruturados, incluíram-se a avaliação qualitativa da sessão, do seu progresso e das técnicas empregadas $(n=11)$, as entrevistas $(n=3)$ e a avaliação por meio de vídeos e áudios $(n=2)$. Na Tabela 1, são apresentados os instrumentos psicométricos que apareceram nas buscas com maior frequência.

\section{Tabela 1}

Frequência dos instrumentos psicométricos mais utilizados

\begin{tabular}{|c|c|c|c|}
\hline Instrumentos & & & $f$ \\
\hline \multirow{4}{*}{ Outcome Questionnaire (OQ-45) } & Outcome Questionnaire OQ- 45 & 24 & \multirow{4}{*}{42} \\
\hline & Outcome Questionnaire OQ-45.2 & 15 & \\
\hline & Outcome Questionnaire OQ 30 & 2 & \\
\hline & Outcome Questionnaire OQ 10.2 & 1 & \\
\hline \multicolumn{2}{|l|}{ Outcome Rating Scale (ORS) } & & 11 \\
\hline \multicolumn{2}{|l|}{ Psychotherapy Process Q-Set (PQS) } & & 8 \\
\hline \multicolumn{2}{|c|}{ Schwartz Outcome Scale-10(SOS-10) } & & 7 \\
\hline \multicolumn{2}{|c|}{ Session Evaluation Questionnaire (SEQ) } & & 5 \\
\hline
\end{tabular}

Como pode ser visto na Tabela 1, o instrumento mais utilizado foi o Outcome Questionnaire (OQ-45), considerando a versão original $(n=21)$, a versão revisada que se trata da OQ-45.2 ( $n=15)$ e a versões reduzidas OQ-30 e OQ-10.2 ( $n=1)$. Além disso, a OQ45 ainda foi utilizada traduzida para o Chile, Espanha, Alemanha e Brasil. O segundo instrumento mais utilizado foi o Outcome Rating Scale-ORS $(n=11)$, com versões em português (Brasil) e em inglês (EUA). Com esses dados a respeito dos instrumentos mais utilizados, foi possível inferir que os estudos na área buscaram realizar a avaliação do processo terapêutico por meio de ferramentas mais estruturadas, ou seja, de cunho 
psicométrico. Considerando essa informação, apresenta-se a seguir a descrição desses instrumentos.

\section{Outcome Questionnaire (OQ-45)}

O OQ-45 foi criado, em 1994, por Lambert, Lunne, Umphress, Hansen e Burlingame, nos EUA, sendo composto por 45 itens a serem respondidos em uma escala Likert de cinco pontos, com o objetivo de avaliar o processo psicoterapêutico, a partir da percepção do cliente. Os itens são agrupados em três fatores, que são: Desconforto subjetivo (aspectos de funcionamento psicopatológico sobre transtornos do humor, ansiedade e uso abusivo de substâncias); Relacionamentos interpessoais (relacionamento familiar, conjugal e amizades) e Desempenho do papel social (aspectos relacionados ao trabalho e ao lazer) (Lambert et al., 1996; Silva, Alves, Peixoto, Rocha, \& Nakano, 2016). Ainda há uma segunda versão da escala, a OQ-45.2, que foi elaborada em 1996, por Lambert, Hansen, Umphress, Lunnen, Okishi, Burlingame e Reisinger, sendo que essa versão manteve a mesma quantidade de itens e fatores que a anterior (Painepán \& Kühne, 2012).

A OQ-45 é, como visto, um dos instrumentos mais utilizados nessa área, sendo traduzido para outras línguas, como: francês, holandês, espanhol, italiano, coreano, japonês, norueguês, sueco, russo e português (do Brasil e Portugal). Diversos estudos têm objetivado verificar as suas propriedades psicométricas, apresentando excelentes indicadores de precisão e validade: $\alpha=0,94$ para o escore total na versão dos EUA; $\alpha=0,90$ na versão brasileira (Boswell, White, Sims, Harrist, \& Romans, 2013; Carvalho \& Rocha, 2009).

\section{Outcome Rating Scale (ORS)}

Desenvolvida por Miller e Duncan (2004) nos Estados Unidos, a ORS tem como objetivo avaliar o progresso do paciente, por meio de autorrelato. É um instrumento curto e pode ser aplicado no início e no final de cada sessão e no pré e pós-tratamento. A avaliação é realizada por meio de quatro dimensões, a saber: Individual (sofrimento ou bem-estar 
pessoal); Interpessoal (dificuldade interpessoal-relacional); Social (a satisfação do paciente com o trabalho/escola e outros ambientes externos); Geral (bem-estar geral). Cada dimensão representa um item que deve ser pontuado numa escala de 0 a 10 . Estudos psicométricos indicaram valores adequados para uso com amostra clínica $(\alpha=0,85)$ e não clínica $(\alpha=0,95)$ (Gillaspy \& Murphy, 2011). Há versão da escala traduzida e adaptada para uso na Noruega (Sundet, 2012), porém não foi encontrado nenhum estudo a respeito da sua tradução e adaptação ao contexto brasileiro e em outros países.

\section{Psychotherapy Process Q-Set (PQS)}

O Psychotherapy Process Q-Set foi criado nos Estados Unidos por Jones (2000), com o intuito de possibilitar a descrição do que acontece no setting terapêutico, no que diz respeito às ações do terapeuta e do cliente, assim como a interação entre eles. Ele é composto por 100 itens que pontuam comportamentos do terapeuta, cliente e da relação entre a dupla. A pontuação é feita por avaliadores externos, a partir da sessão psicoterápica transcrita literalmente ou gravada. Os itens, apresentados em cartões, são analisados em relação uns com os outros e não de maneira independente, sendo ordenados de acordo com o observado em sessão. Os itens devem ser dispostos em nove pilhas que constituem uma escala que vai do menos característico ao mais característico em relação à sessão analisada. É necessário treinamento para pontuação desse instrumento, tendo em vista que os avaliadores deverão considerar frequência, intensidade e relevância de cada item em relação aos demais, a fim de ordená-lo. Dessa forma, esse processo requer conhecimento de todos os itens, reflexão sobre a influência de um item sobre o outro, além da apreciação total da sessão avaliada (Jones, 2000).

O instrumento foi validado para uso em outros países, como Alemanha, Japão, Itália, Espanha, Noruega e Brasil (Ablon, Levy, \& Smith-Hansen, 2011; Nardi, Serralta, \& Benetti, 2016). Estudos de adaptação e evidências de validade para o Brasil foram conduzidos, indicando ser adequado para uso. A confiabilidade entre avaliadores em todos os 100 itens do PQS produziu coeficientes alfa entre 0,83 e 0,89 e as análises de confiabilidade também apresentaram valores entre aceitáveis e excelentes (entre 0,50 e 0,95) (Ablon et al., 2011). 
No Brasil, o instrumento traduzido por Serralta et al. (2007) apresentou boa equivalência semântica com o instrumento original e fidedignidade entre os avaliadores variando entre 0,53 e 0,72 .

\section{Schwartz Outcome Scale-10 (SOS-10)}

A SOS-10 foi criada nos Estados Unidos por Blais et al. (1999). É uma escala composta por dez itens que têm por objetivo avaliar o bem-estar psicológico da pessoa durante a semana que passou após o atendimento. A avaliação é realizada por uma escala Likert de sete pontos, na qual o respondente deve dizer como se sentiu na última semana em diferentes aspectos, como, por exemplo, relacionamentos, futuro, vida pessoal, diversão, entre outros. Quanto maior a pontuação, melhor foi a avaliação do cliente em relação aos resultados obtidos.

A escala ainda não foi validada no Brasil. No entanto, além dos Estados Unidos, há estudos de validação no Canadá, Espanha, Kuwait e República Tcheca. Os estudos de validade indicaram que se trata de uma escala unidimensional e com valores de confiabilidade que variam entre 0,96 e 0,79, a depender do país. A SOS-10 se correlacionou com medidas de depressão e bem-estar psicológico e se mostrou sensível às mudanças ocorridas durante o processo psicoterapêutico (Laux, Young, McLaughlin, \& Perera-Diltz, 2006; Mohammed, 2008; Rivas-Vazquez et al., 2001).

\section{Session Evaluation Questionnaire (SEQ)}

Esta escala foi desenvolvida nos Estados Unidos por Stiles, em 1980, para avaliar a percepção do cliente quanto à profundidade e qualidade das sessões, sendo que ela também possui uma versão voltada para o terapeuta. A SEQ é composta por 21 pares de adjetivos bipolares, distribuídos em uma escala Likert de sete pontos. Os itens são divididos em duas dimensões, assim, o respondente deve assinalar sobre sua percepção da sessão e também seu estado de humor. As dimensões são classificadas entre poderoso/fraco, valioso/sem valor, profundo/superficial, especial/normal e cheia/vazia. Assim, as pontuações mais altas, 
ou seja, mais próximas a sete indicam uma percepção da sessão mais intensa (Hafkenscheid, 2008).

Uma análise dos fatores do instrumento indicou duas dimensões para a avaliação da sessão, chamadas de profundidade e suavidade, assim como duas dimensões para o momento pós-sessão, rotuladas como positividade e excitação. Há ainda duas versões do instrumento, uma aprofundada e a outra superficial (Hafkenscheid, 2008). No estudo de Stiles et al. (1994), verificou-se um $\alpha=0,90$ que é considerado adequado, ou seja, a escala é apropriada para uso. Desde a sua criação, o SEQ foi traduzido para diversos idiomas, como: francês, alemão, grego, chinês, tailandês e holandês (Hafkenscheid, 2008). Até o momento, não foi verificada uma versão traduzida para o Brasil.

\section{Discussão}

Este estudo teve como objetivo mapear instrumentos que avaliam a percepção de eficiência do processo psicoterapêutico, tanto no contexto nacional como internacional, sendo que foram encontrados 192 instrumentos em diferentes estudos. Percebe-se, a partir disso, que a avaliação do processo psicoterapêutico é um tema que tem sido muito estudado por meio de distintas formas de avaliação e que há uma preocupação cada vez maior de pesquisadores de diversas nacionalidades e instituições em avaliar se os mesmos estão sendo realmente eficazes (Brum et al., 2012; Nardi et al., 2016).

Isso fica claro ao verificar o aumento do número de estudos ao longo dos anos, principalmente após 2009. Uma provável explicação para esse aumento está relacionada à necessidade de adoção de técnicas e procedimentos psicoterápicos que sejam realmente eficazes, reduzindo, dessa forma, tempo e custos com cada paciente. Ao se avaliar quais os melhores tipos de intervenção e seus resultados ao longo do processo, é possível beneficiar um maior número de pacientes em um menor espaço de tempo, direcionando os custos investidos na área de maneira mais racional. Dessa forma, o aumento da produção reflete essa preocupação com saúde pública, tanto em nível nacional quanto internacional (Couto, 2015; Drozd \& Goldfried, 1996; Duncan, 2012; Peuker et al., 2009). 
No que diz respeito à nacionalidade dos autores, percebe-se que os Estados Unidos ficaram em primeiro lugar e que o Brasil ficou em segundo lugar ao se verificar todas as publicações. No entanto, mesmo o Brasil ficando em segundo lugar, somente nove estudos foram encontrados no levantamento realizado, o que contrasta com as 106 pesquisas na área realizadas nos EUA. Esse achado é coerente com outras pesquisas na área da saúde, em que os EUA têm destaque em quantidade de produção. Como parte da busca, foram utilizadas palavras em português e, atendendo aos objetivos de verificar também a produção nacional, compreende-se que foram resgatados mais estudos brasileiros. Apesar disso, deve-se ressaltar que a maioria dos periódicos usa o inglês como segunda língua, o que demonstra que muito provavelmente outros países não atingiram tantas publicações quanto o Brasil nessa temática. Esse aspecto poderia ser mais bem averiguado ao se acrescentar as palavras-chave em mais algum idioma, por exemplo, o espanhol.

Um importante dado referente aos instrumentos localizados é a variedade de idiomas disponíveis para um mesmo instrumento. Por exemplo, a OQ-45, Outcome Rating Scale, Psychotherapy Process Q-Set, Schwartz Outcome Scale-10 e Session Evaluation Questionnaire, inicialmente desenvolvidos por estadunidenses (Blais et al., 1999; Miller \& Duncan, 2004; Silva et al., 2016; Stiles et al., 1994), apresentam traduções e estudos de evidências de validade em outros países, como, por exemplo, Alemanha, Brasil, República Tcheca, Holanda, Japão, Itália, Kuwait, Noruega e Espanha. A maioria dos instrumentos, mesmo alguns semiestruturados ou não estruturados contou com estudos psicométricos, indo de acordo também com a tendência internacional de avaliar as qualidades psicométricas (AERA, APA, \& NCME, 2014; Brum et al., 2012; Peuker et al., 2009). Todos eles verificaram a confiabilidade dos respectivos instrumentos, encontrando suficientes evidências dentro da especificidade de cada um e para os respectivos países.

Outra característica que foi possível notar, ao se comparar os cinco instrumentos que apareceram com maior frequência, foi a preocupação com o desenvolvimento de ferramentas mais estruturadas, ou seja, de cunho quantitativo. Mesmo o Brasil, que até recentemente produzia mais instrumentos qualitativos como entrevistas e crivos de observação (Silveira \& Wiethaeuper, 2003), passou mais fortemente, a partir de 2005, a adaptar e desenvolver instrumentos quantitativos, o que pode ter sido estimulado pelo 
desenvolvimento das técnicas estatísticas (Pasquali, 2017). Além disso, a busca por ferramentas mais estruturadas, em contraponto às semiestruturadas/não estruturadas cuja característica principal é o método qualitativo, atende à necessidade de instrumentos com menor viés de aplicador, favorecendo aplicação padronizada, sem necessidade de treinamentos extensos e, consequentemente, custosos.

Os dados encontrados neste estudo estão de acordo com as pesquisas de Serralta et al. (2007) e Yoshida et al. (2005), que já discutiam a questão de um delineamento mais quantitativo em estudos internacionais. Embora tenha ocorrido um aumento da produção e adaptação de instrumentos quantitativos, compreende-se que essa ainda seja uma lacuna a ser trabalhada no contexto brasileiro (Brum et al., 2012; Couto, 2015; Silveira \& Wiethaeuper, 2003).

No que diz respeito especificamente à adaptação para o Brasil das medidas de avaliação mapeadas nesta revisão, percebe-se que entre os cinco instrumentos mais frequentemente relatados em pesquisas, o OQ-45 e o Psychotherapy Process Q-Set foram adaptados para uso no Brasil (Boswell et al., 2013; Carvalho \& Rocha, 2009; Serralta, Nunes, \& Eizirik, 2007). Isso pode indicar um movimento de pesquisadores brasileiros no sentido de desenvolver mais pesquisas na área, inclusive em diálogo com a literatura internacional, indicando, também, maior atenção às particularidades da avaliação do processo psicoterapêutico.

Apesar disso, nota-se que tal movimento ainda vem ocorrendo lentamente, visto que, dentre os cinco instrumentos, apenas dois foram traduzidos e adaptados. Isso pode estar relacionado tanto à forma quanto à temática, bem como à necessidade de instrumentos para verificar a eficácia dos processos psicoterapêuticos, principalmente no Brasil. Ou seja, é relativamente recente a preocupação dos pesquisadores com essas questões. Logo, o trabalho de adaptação desses instrumentos para o Brasil também é incipiente. Assim, verificar se e como pesquisadores brasileiros estão estudando formas de avaliação da psicoterapia, não apenas por seus resultados, mas enquanto processo, é algo que deve ser analisado em pesquisas futuras, a fim de verificar se esse panorama se mantém ao longo do desenvolvimento na área e de que forma. 
Outro ponto a ser discutido, embora apenas tangencial ao objetivo desta pesquisa, concerne à fundamental importância para a prática em avaliação psicológica: o treinamento de profissionais na administração das ferramentas. Como foi apresentado, alguns instrumentos são semiestruturados, como por exemplo, entrevistas, observações e envolvem participação mais ativa do avaliador. É consenso na literatura a necessidade de adequado treinamento de estudantes e profissionais ao conduzir qualquer prática terapêutica, incluindo a avaliação do processo psicoterapêutico (Baptista, 2010; Borsa, 2016). Nenhum estudo focou nesse quesito, embora os que envolviam técnicas semiestruturadas tenham apresentado esta característica para análise. Ainda assim, para uso em pesquisas futuras ou mesmo para a adaptação das técnicas para o Brasil, seria necessário o desenvolvimento de check-lists ou treinamentos para condução das administrações de forma que elas sejam realizadas sempre da mesma forma, buscando um padrão nas avaliações.

\section{Considerações finais}

Dado o exposto, é importante ressaltar que esta pesquisa visou buscar somente instrumentos que avaliassem o processo psicoterapêutico em si, não considerando neste levantamento as pesquisas que avaliam a eficácia terapêutica com base na redução dos sintomas medidos antes e depois de uma intervenção e que utilizaram instrumentos que não foram desenvolvidos especificamente para avaliação da eficácia do tratamento, como, por exemplo, medidas de qualidade de vida, depressão, ansiedade, entre outras possibilidades. Ou seja, não foram incluídos estudos que usaram instrumentos como escalas de sintomas, uma vez que estes não medem efetivamente a eficiência do processo psicoterapêutico e que a melhora de um sintoma, dentro de um processo todo, pode ser pouco para atestar sua eficiência. Conforme Brum et al. (2012), essa distinção é importante, uma vez que há enfoques específicos para cada área. A redução de sintomas focando mais em instrumentos que mensurem características específicas de um grupo clínico, enquanto o processo centrase nos elementos importantes para o desenvolvimento do tratamento psicoterapêutico.

Assim, o que foi observado em termos de produtividade diz respeito apenas aos estudos que focam o processo psicoterapêutico, por considerarem a remoção em si de um 
sintoma menos relevante do que a experiência como um todo e o desenvolvimento pessoal que ela propicia. Certamente, outras pesquisas poderão abarcar também a perspectiva voltada para sintomas, fomentando uma discussão sobre critérios de eficácia terapêutica (Brum et al., 2012).

Além disso, vale ressaltar que este estudo também não se propôs a avaliar o quanto as medidas ora encontradas são realmente eficientes/sensíveis para avaliação do processo, tampouco a sua relação com outros instrumentos e construtos. O que se observou, conforme mencionado anteriormente, é que os instrumentos apresentaram evidências de validade e precisão (AERA et al., 2014; Brum et al., 2012; Peuker et al., 2009) verificadas por meio de estudos psicométricos. A análise dessa questão é complexa, sendo necessário computar os valores de validade e precisão encontrados em cada estudo e em cada ferramenta e, assim, verificar sistematicamente a sensibilidade de cada instrumento em avaliar aquilo que se propõem.

Em estudos futuros, essa questão poderia ser desenvolvida por meio de metanálises que tenham como proposta principal verificar as propriedades psicométricas/sensibilidade dos instrumentos de avaliação do processo psicoterapêutico. Por fim, esta revisão permitiu levantar quais são os instrumentos que estão sendo usados com maior frequência nas pesquisas sobre o processo terapêutico e, a partir disso, o que pode vir a contribuir para o desenvolvimento de estratégias terapêuticas ou procedimentos de intervenção mais exitosos, ou ainda propiciar uma reflexão sobre a formação e o treinamento de novos profissionais, com base nos estudos.

\section{Referências}

Ablon, J. S., Levy, R. A., \& Smith-Hansen, L. (2011). The contributions of the psychotherapy process Q-set to psychotherapy research. Research in Psychotherapy, 14(1), 14-48. https://doi.org/10.4081/ripppo.2011.46

AERA, A. E. R. A., APA, A. P. A., \& NCME, N. C. on M. in E. (2014). Standards for educational and psychological testing. Washington: American Educational Research Association.

Arnow, B. A., Steidtmann, D., Blasey, C., Manber, R., Constantino, M. J., Klein, D. N. et al. (2013). The relationship between the therapeutic alliance and treatment outcome in two 
distinct psychotherapies for chronic depression. Journal of Consulting and Clinical Psychology, 81(4), 627-638. https://doi.org/10.1037/a0031530

Baptista, M. N. (2010). Questões Sobre Avaliação de Processos Psicoterápicos. Psicologia em Pesquisa, 4(2), 109-117. http://pepsic.bvsalud.org/pdf/psipesq/v4n2/v4n2a04.pdf

Blais, M. A., Lenderking, W. R., Baer, L., deLorell, A., Peets, K., Leahy, L. et al. (1999). The Schwartz Outcomes Scale-10: A brief measure of psychological well-being. Journal of Personality Assessment, 73(3), 359-373.

Borsa, J. C. (2016). Considerações sobre a formação e a prática em avaliação psicológica no Brasil. Temas em Psicologia, 24(1), 131-143. https://doi.org/10.9788/TP2016.1-09

Boswell, D. L., White, J. K., Sims, W. D., Harrist, R. S., \& Romans, J. S. (2013). Reliability and validity of the Outcome Questionnaire-45.2. Psychological Reports, 112(3), 689-693. https://doi.org/10.2466/02.08.PR0.112.3.689-693

Brum, E. H. M., Frizzo, G. B., Gomes, A. G., Silva, M. da R., Souza, D. D., \& Piccinini, C. A. (2012). Evolução dos modelos de pesquisa em psicoterapia. Estudos de Psicologia (Campinas), 29(2), 259-269. https://doi.org/10.1590/S0103-166X2012000200012

Carvalho, L. F., \& Rocha, G. M. A. (2009). Tradução e adaptação cultural do Outcome Questionnaire (OQ-45) para o Brasil. Psico-USF, 14(3), 309-316. https://doi.org/10.1590/S1413-82712009000300007

Cassel, P. A., Sanchez, L. F., Campezatto, P. M., \& Nunes, M. L. T. (2015). Processo psicoterapêutico: compreensão de momentos de mudança psicológica em uma sessão de psicoterapia psicanalítica. Contextos Clínicos, 8(1), 27-37. http://doi.org/10.4013/ctc.2015.81.03

Couto, P. M. (2015). Avaliação da eficácia de um programa de interveção junto de mulheres alcoólicas [Dissertação de mestrado, Universidade Fernando Pessoa]. Repositório Institucional da Universidade Fernando Pessoa.

Drozd, J. F., \& Goldfried, M. R. (1996). A critical evaluation of the state-of-the-art in psychotherapy outcome research. Psychotherapy, 33(2), 171-180. https://doi.org/10.1037/0033-3204.33.2.171

Duncan, B. L. (2012). The partners for change outcome management system (PCOMS): The heart and soul of change project. Canadian Psychology, 53(2), 93-104. https://doi.org/10.1037/a0027762

Ferreira, N. S., \& Yoshida, E. M. P. (2004). Produção científica sobre psicoterapias breves no Brasil e demais países latino-americanos (1990-2000). Estudos de Psicologia (Natal), 9(3), 523-531. http://doi.org/10.1590/S1413-294X2004000300015 
Gillaspy, J. A., \& Murphy, J. J. (2011). Incorporating outcome and session rating scales in Solution-focused Brief Therapy. In H. Ratnet, C. Iveson, \& E. George (Orgs.), Solution Focused Brief Therapy: 100 Key Points and Techniques (pp. 73-91). New York: Oxford University Press.

Hafkenscheid, A. (2008). The impact of psychotherapy sessions: Internal structure of the Dutch Session Evaluation Questionnaire (SEQ). Psychology and Psychotherapy Theory Research and Practice, 82, 99-111. https://doi.org/10.1348/147608308X380750

Harker, J., \& Kleijnen, J. (2012). What is a rapid review? A methodological exploration of rapid reviews in Health Technology Assessments. International Journal of Evidence-Based Healthcare, 10, 397-410. https://doi.org/10.1111/j.1744-1609.2012.00290.x

Jones, E. E. (2000).Therapeutic action: a guide to psychoanalytic therapy. Northvale: Aronson.

Lambert, M. J., Burlingame, G. M., Umphress, V., Hansen, N. B., Vermeersch, D. A., Clouse, G. C. et al. (1996). The Reliability and Validity of the Outcome Questionnaire. Clinical Psychology \& Psychotherapy, 3(4), 249-258. https://doi.org/10.1002/(SICl)10990879(199612)3:4<249::AID-CPP1 06>3.0.CO;2-S

Laux, J. M., Young, J. A., McLaughlin, L., \& Perera-Diltz, D. (2006). The development of a French version of the SOS-10 (SOS-10-F). Canadian Journal of Counselling, 40, 195208. https://www.learntechlib.org/p/71969/

Lutz, W., De Jong, K., \& Rubel, J. (2015). Patient-focused and feedback research in psychotherapy: Where are we and where do we want to go? Psychotherapy Research, 25(6), 625-632. https://doi.org/10.1080/10503307.2015.1079661

Miller, S. D., \& Duncan, B. L. (2004). The Outcome and Session Rating Scales: Administration and scoring manual. Chicago: Institute for the Study of Therapeutic Change.

Mohammed, H. R. (2008). Development of an Arabic Version of the Schwartz Outcome Scale10: A Brief Mental Health Outcome Measure. Journal of Educational \& Psychological Sciences, 9(3), 11-24. http://doi.org/10.12785/JEPS/090311

Nardi, S. C. S., Serralta, F. B., \& Benetti, S. P. C. (2016). Uso do psychotherapy process Q-set para pesquisas em psicoterapias psicanalíticas: revisão sistemática da literatura. Estudos e Pesquisas em Psicologia, 16(1), 220-239. https://doi.org/10.12957/epp.2016.24840

Painepán, B., \& Kühne, W. (2012). Efectividad según duración de la psicoterapia en un centro de atención psicológica para universitarios. Summa Psicológica, 9(1), 47-52. http://pepsic.bvsalud.org/pdf/summa/v9n1/a05.pdf

Pasquali, L. (2017). Psicometria: Teoria dos testes na psicologia e na educação. Petrópolis: Editora Vozes. 
Peuker, A. C., Habigzang, L. F., Koller, S. H., \& Araujo, L. B. (2009). Avaliação de processo e resultado em psicoterapias: uma revisão. Psicologia em Estudo (Maringá), 14(3), 439445. http://doi.org/10.1590/S1413-73722009000300004

Rivas-Vazquez, R., Rivas-Vazquez, A., Blais, M. A., Rey, G. J., Rivas-Vazquez, F., \& Jacobo, M. (2001). Development of a Spanish Version of the Schwartz Outcome Scale-10: A Brief Mental Health Outcome Measure. Journal of Personality Assessment, 77(3), 436-446. https://doi.org/10.1207/S15327752JPA7703_05

Seligman, M. E. P. (1995). The effectiveness of psychotherapy: The consumer reports study. American Psychologist, 50(12), 965-974. https://doi.org/10.1037/0003066X.50.12.965

Serralta, F. B., Nunes, M. L. T., \& Eizirik, C. L. (2007). Elaboração da versão em português do Psychotherapy Process Q-Set. Revista de Psiquiatria, 29(1), 44-55. http://doi.org/10.1590/S0101-81082007000100011

Silva, S. M., Alves, I. C. B., Peixoto, E. M., Rocha, G. M. A., \& Nakano, T. C. (2016). Outcome Questionnaire (OQ-45.2): avaliação das propriedades psicométricas via modelo bifactor e TRI. Psico (Porto Alegre), 47(4), 298-308. https://doi.org/10.15448/1980$\underline{8623.2016 .4 .24600}$

Silveira, A. M., \& Wiethaeuper, D. (2003). Considerações em torno das atuais correntes predominantes da pesquisa em psicoterapia. Revista Brasileira de Psicoterapia, 5(1), 3352.

Stiles, W. D., Reynolds, S., Hardy, G. E., Rees, A., Barkham, M., \& Shapiro, D. A. (1994). Evaluation and description of psychotherapy sessions by clients using the Session Evaluation Questionnaire and the Session Impacts Scale. Journal of Counseling Psychology, 41, 175-185.

Sundet, R. (2012). Therapist perspectives on the use of feedback on process and outcome: Patient-focused research in practice. Canadian Psychology, 53(2), 122-130. http://doi.org/10.1037/a0027776

Yoshida, E. M. P., Santeiro, T. V., Moraes, F. R., \& Rocha, G. M. A. (2005). Psicoterapias breves psicodinâmicas: características da produção científica nacional e estrangeira (1980/2003). Psico-USF, 10(1), 51-59. http://doi.org/10.1590/S141382712005000100007 